In press at Current Opinion in Psychology.

This paper is not the copy of record and may not exactly replicate the final,

authoritative version of the article.

The Relation of Mindfulness and Prosocial Behavior: What Do We (Not) Know?

Simon Schindler ${ }^{1}$, Malte Friese ${ }^{2}$

${ }^{1}$ Dresden University of Technology, Germany; ${ }^{2}$ Saarland University, Germany

Corresponding author:

Simon Schindler

Dresden University of Technology

Faculty of Psychology

BZW A209, Zellerscher Weg 17

01069 Dresden, Germany

simon.schindler@tu-dresden.de 


\begin{abstract}
Mindfulness is a hot topic in psychological research and the popular media. One central claim in the literature is that enhanced mindfulness fosters prosocial behavior. This article recapitulates what is currently known about this widespread claim. We first review theoretical perspectives on why enhanced mindfulness should foster prosocial behavior and discuss relevant empirical evidence. Two meta-analyses provide preliminary support for this claim. However, limitations call for caution when interpreting the evidence and studies investigating effects that persist over sustained periods of time are missing. In addition, theoretical assumptions about the underlying mechanisms need stronger empirical support. We discuss theoretical predicaments, identify potential downsides of mindfulness, and suggest ways forward for future research.
\end{abstract}

112 words

Keywords: Mindfulness; prosocial behavior; self-regulation; empathic concern; motivation

\title{
Highlights
}

- Enhanced mindfulness is assumed to increase prosocial behavior

- We review the relevant empirical evidence

- Two meta-analyses provide tentative support for the claim

- Theoretical and empirical issues dampen the conclusiveness of the literature

- We suggest ways forward 
Mindfulness is a hot topic in both psychological research and the popular media. One reason for this popularity lies in the presumed broad beneficial consequences of being mindful for mental and physical health [1]. One widespread assumption is that mindfulness not only benefits the self, but also others. In the present article, we review theoretical assumptions arguing that increased mindfulness leads to more prosocial behavior and survey empirical evidence relevant to this claim. Special attention relies on evidence for sustained changes in prosocial behavior as a function of changes in mindfulness due to their arguably great relevance concerning real-world implications of this research. We critically reflect on the foundational theoretical assumptions and the empirical evidence and suggest future research directions.

\section{Conceptual clarifications}

1.1 Mindfulness. There is an ongoing debate on the precise definition of mindfulness $[2,3]$. Some consensus exists on conceptualizing mindfulness as an open and nonjudgmental awareness of one's present-moment experiences [4-6]. Specifically, mindfulness includes meta-cognitive awareness of one's internal mental and emotional experiences, observing them as such, and watching them come and go. That is, mindful persons take the position of neutral, nonjudging observers of their thoughts and emotions, without reflecting on their valence or content. Mindful persons are assumed to recognize experiences without ruminating about, identifying with or judging them. As a state of present-moment awareness, mindfulness is often described as the opposite of unintentional mind wandering [7] or acting on "autopilot" [8].

Mindfulness varies as a stable trait between persons (i.e., some people are more mindful than others across situations), but it also varies as a state across time within persons (i.e., a person is sometimes mindful and sometimes not). Researchers attempt to measure both traits and states, and they also seek to experimentally manipulate mindfulness on both levels [9]. The dominant way to cultivate mindfulness is meditation. However, there are many 
meditation practices, and all differ in focus and instructions (e.g., loving kindness, forgiveness, compassion, generosity) implying different theoretical assumptions. To secure high internal validity, it is important to distinguish mindfulness meditation from other meditation practices when reviewing the literature [10]. Mindfulness has its roots in Buddhist contemplative practices, but most scientific studies on mindfulness are secular and, in contrast to other meditation practices, lack grounding in explicit ethics-based instructions found in Buddhist traditions [11].

1.2 Prosocial behavior. Prosocial behavior can be broadly defined as any action that benefits another [12]. There are different underlying motivations of prosocial behavior: For example, feelings of empathic concern promote prosocial behavior with the primary goal of improving the welfare of the person in need and without expectation of personal reward (other-oriented, altruistic motivation; [13]). Prosocial behavior can also result from non-altruistic motivations such as conforming to social norms (e.g., reciprocity or justice; $[14,15])$ and/or self-oriented (egoistic) motivation, for instance when aiming to reduce aversive arousal and negative affect, such as feelings of guilt $[16,17]$.

\section{Mindfulness and prosocial behavior: Theoretical Assumptions}

Surveying the mindfulness literature reveals several reasons why an increase in mindfulness should lead to an increase in prosocial behavior. We identified two prominent lines of arguments. First, mindfulness is assumed to be linked to enhanced executive control and the promotion of self-regulatory capacity [9,18,19]. Accordingly, it is argued that higher mindfulness improves the ability to direct and sustain attention. Improving this ability should increase awareness of others' needs in the social environment $[20,21 * *]$. It is further argued that — because mindfulness is believed to foster executive functions including inhibitionnegative emotions (such as fear of failing or disgust) can be more effectively regulated and overcome in a helping situation when the person is in a mindful state. Consequently, 
mindfulness should promote prosocial behavior especially when such negative emotions may be present $[22 * *]$.

The second line of argument refers to the assumption that an increase in mindfulness leads to higher empathic concern $\left[21^{* *}, 22^{* *}\right]$. Empathic concern (or "compassion") can be defined as feelings that are elicited in response to suffering others [23]. Mindfulness interventions typically facilitate disengaging from and disidentifying with mental contents by meeting them with nonjudgmental acceptance [4]. Training such an ability is assumed to attenuate self-referential thoughts and emotions, further reducing boundaries between the self and others and in turn increasing empathic concern for others in need [22**]. Given that prosocial behavior is often motivated by empathic concern, increased mindfulness is argued to increase prosocial behavior.

\section{Does mindfulness increase prosocial behavior? Empirical Evidence}

3.1 Correlational evidence. When reviewing evidence on the effects of mindfulness on prosocial behavior, it is important to distinguish between correlational studies that include mindfulness as a trait and studies that included an (experimental) intervention $[3,10]$. Correlational studies necessarily remain inconclusive with respect to causal evidence; only experimental studies (sometimes called randomized controlled trials; RCTs) allow for causal conclusions.

A recent meta-analysis investigated the relationship between trait mindfulness and prosocial outcomes [21*]. Across 32 effect sizes from twelve studies, trait mindfulness was positively associated with prosocial outcomes, $d=0.73,95 \%$ CI $[0.51,0.96]$. Noticeably, the effects sizes distributed across a wide spectrum, ranging from less than zero to about $d=1.60$, twice the size of a conventionally large effect [24]. When focusing on observer-reported (instead of self-reported) prosocial behaviors, the association was considerably weaker, $d=$ $0.37,95 \%$ CI [0.19, 0.79] (see also [25]). 
The applied tests did not reveal evidence for publication bias (but modern approaches were not applied; [26]). The interpretation of the evidence was somewhat complicated by the heterogeneity of the included trait mindfulness scales [3] and the high prevalence of selfreports to assess prosocial outcomes that are prone to contamination by social desirability bias.

3.2 Experimental evidence. We discuss two relevant meta-analyses that included RCTs. ${ }^{1}$ In their meta-analysis, Donald et al. [21**] found a positive effect of mindfulness-based interventions on prosocial outcomes, $d=0.51,95 \% \mathrm{CI}[0.37,0.66]$ that was based on 21 studies and 41 effect sizes. Neither intervention type (mindfulness-only or mindfulness-plus an additional component) or type of outcome (observer vs. self-reported behaviors) moderated this effect. Effects were similarly strong for brief interventions lasting less than one hour and interventions lasting over 10 hours.

The conclusiveness of the evidence was somewhat curtailed by evidence for publication bias (Eggers' test and stronger effects for published than unpublished studies). In addition, the arguably most conservative and internally valid analysis of the data was not reported: an analysis exclusively based on mindfulness-only interventions and observer-rated prosocial behavior.

The preregistered meta-analysis by Berry et al. [22**] provides a stronger test of the claim that mindfulness increases prosocial behavior. Here, the authors included (a) only RCTs that (b) featured mindfulness-only interventions, and (c) assessed overt (but not self-reported) prosocial behavior. Notably, the authors interpreted attenuated antisocial behavior (reducing prejudice and retaliation) as a type of prosocial behavior. Analyzing the 29 effect sizes yielded a small-to-medium positive effect of mindfulness interventions, $g=0.43,95 \% \mathrm{CI}$ $[0.30,0.55]$. Follow-up analyses revealed significant effects on compassionate helping

\footnotetext{
${ }^{1}$ We do not refer to the meta-analyses by Kreplin et al. [27] and Luberto et al. [28] because most of the included studies applied meditation types that were not specific to mindfulness (e.g., loving-kindness).
} 
behavior, $g=0.55,95 \%$ CI $[0.45,0.65]$, reducing prejudice, $g=0.46,95 \%$ CI $[0.30,0.63]$, reducing retaliation, $g=0.54,95 \% \mathrm{CI}[0.29,0.78]$, and instrumental helping, $g=0.27,95 \%$ CI $[0.02,0.53]$. Effects on generosity were not significant, $g=0.03,95 \%$ CI $[-0.16,0.23]$. Again, there was evidence for publication bias (Egger's test); a trim-and-fill analysis suggested an adjusted effect size of $g=0.32$, a reduction by about $25 \%$. A $p$-curve analysis (based on 15 significant $p$-values) suggested evidential value in the set of included studies but also indicated a low average power of $31 \%(90 \%$ CI $[8 \%, 62 \%])$, suggesting that it is most likely that only $31 \%$ of the studies would replicate. Of all included studies, only one study was preregistered, and this particular study showed no effect.

Of note, compassion-related behaviors (e.g., offering a seat to a person in need, writing a comforting email to an ostracized person) seem more sensitive to mindfulness interventions than generosity-related behaviors. This is in line with the assumption that increased mindfulness increases empathic concern (a construct similar to "compassion"). However, compassion-related behaviors were typically assessed during the experimental session and did not entail any further costs on the part of the participants (in terms of money or time). By contrast, generosity-related behaviors typically entailed costs (e.g., money for donations). For this latter type of prosocial behavior, Berry et al. [22**] found no reliable meta-analytic effect of mindfulness interventions. The question thus remains, whether increased mindfulness increases costly prosocial behavior (see [25]).

3.3 Limited conclusiveness. Taken together, recent meta-analyses are consistent with the claim that enhanced mindfulness increases prosocial behavior. The conclusiveness of these findings is limited due to the relatively small number of high-quality experimental studies. For example, many primary studies had remarkably small sample sizes that are unable to reliably detect effects of realistic magnitudes [29] and few studies included manipulation checks. This is problematic given the high heterogeneity of mindfulness interventions regarding their instructions and duration (a few minutes vs. days vs. weeks). In addition, there 
is evidence for publication bias that can distort meta-analytic effect size estimates [30].

Modern approaches to correct for publication bias have not yet been applied to this literature $[26]$.

To substantiate the idea that effects of increased mindfulness on prosocial behavior have meaningful real-world implications, it is essential to provide evidence for effects that (a) persist over sustained periods of time, and (b) emerge on a broad range of expressions of prosocial behavior. Unfortunately, it is precisely this kind of evidence that is sorely missed. Most studies measured the prosocial outcome(s) immediately or shortly after the end of training and did not include follow-ups to assess stability of effects. In addition, most studies employed one-shot behavioral measures targeting one specific expression of prosocial behavior only. However, even people dispositionally high in mindfulness are not mindful all the time. Similar to other traits, mindfulness markedly varies within individuals across time [31]. This means that we cannot expect a disposition to be mindful to reliably predict behavior in singled out situations [32]. Instead, we need evidence that covers multiple assessments across longer periods of time.

To be sure, we do not insinuate that intervention studies that assess their dependent measure shortly after the intervention and that use one-shot dependent measures are not worth the effort. These studies can provide valuable proof-of-principle evidence (i.e., they can show that in principle a mindfulness intervention can at least briefly foster prosocial behavior). However, to confidently reinforce the standpoint that mindfulness can foster prosocial behavior in ways that matter for everyday life, we need more compelling evidence. One way to tackle this challenge is to implement (potentially repeated) experience sampling periods before and after mindfulness interventions. Experience sampling would allow researchers to obtain a reliable impression of effects on a broad range of prosocial behaviors in daily life across prolonged time periods. It would also allow to more conclusively investigate the 
mental processes presumably triggered by mindfulness interventions and responsible for effects on prosocial behavior (e.g., empathic concern).

\section{Boosting theorizing: When does mindfulness (not) increase prosocial behavior?}

Besides grappling with the empirical evidence, it seems fruitful to reconsider why increased mindfulness should promote prosocial behavior. We outlined two prominent lines of theoretical arguments: First, increased mindfulness is linked to improved executive control and self-regulation abilities. Second, mindfulness increases empathic concern. The idea that increased mindfulness is positively linked to empathic concern may be rooted in anecdotal reports and the Buddhist origin of mindfulness. So far, however, there is little evidence for a direct positive effect of increased mindfulness on empathic concern or feelings of compassion [18,33]. Again, it is crucial to differentiate between the types of meditation regarding additional prosocial components in the instructions [34].

The second line of argument refers to the assumption that increased mindfulness is positively linked to improved executive control and self-regulatory abilities, which should direct and sustain attention to others' needs and inhibit negative emotions. Compared to the argument of increased empathic concern, this assumption received more empirical support $[18,19]$, but high-quality studies are rare [9]. Typical mindfulness exercises contain explicit instructions to focus on one's breath and to take the position of a neutral, nonjudging observer of one's own emotions and thoughts. It seems plausible that such exercises improve the ability to direct and sustain attention, to recognize impulses and emotions without further ruminating and finally to down-regulate them. However, the psychological processes underlying the effects of mindfulness on self-regulatory behavior are not fully understood. One preliminary interpretation of the literature is that mindfulness may be regarded as a tool that can help to attain self-regulatory goals, but that does not necessarily foster restraint [9].

Even if increased mindfulness would actually improve self-regulation abilities, it is not clear why this should automatically result in increased prosocial behavior. Regarding the 
assumed underlying motivations (other or self-oriented motivations) of prosocial behavior, there is no direct link between self-regulation and increased prosocial behavior. In fact, mindfulness represents an observational and receptive state, rather than a motivational state [35]. From this perspective, mindfulness does not automatically lead to prosocial behavior-a resulting prosocial motivation should depend on other factors, such as personal salient goals. In line with this idea, recent evidence suggests that the effect of mindfulness interventions differ by how participants construe themselves [36*]. In two studies, mindfulness meditation increased prosocial behavior among participants with self-reported interdependent selfconstruals or primed with interdependence but led to decreased prosocial behavior among participants with self-reported independent self-construals or primed with independence. To explain their findings, the authors refer to the assumption that increased mindfulness increases self-awareness and self-clarity instead of reducing ego-involvement and self-referential thoughts.

So far, scholars mostly investigated effects of mindfulness on prosocial behavior by relying on other-oriented motivation of prosocial behavior. However, prosocial behavior is often based on a self-oriented motivation, such as the aim to reduce negative states and aversive feelings. Assuming enhanced mindfulness to attenuate such feelings (see [9]), enhanced mindfulness can also be expected to lead to less prosocial behavior. In line with this notion, across five studies, Schindler et al. [37*] found that a brief mindfulness intervention attenuated repairing behavior that usually results from a bad conscience when having caused harm. These results point to a potential downside of mindfulness by attenuating automatic prosocial reactions based on negative emotions (such as guilt), suggesting that mindfulness is not inherently pro- or anti-social—at least in the Western world where mindfulness practices lack grounding in ethics-based instructions.

\section{Conclusions}


Recent meta-analyses are consistent with the idea that mindfulness increases prosocial behavior. Although encouraging, the evidence is still preliminary. More high-powered, highquality studies are needed that provide causal evidence that persists over sustained periods of time on a broad range of expressions of prosocial behavior. This kind of evidence would go a long way to substantiate the great expectations concerning real-world implications of the effects of mindfulness on prosocial behavior. In addition, the adherence to standards of open, transparent science would increase the confidence in the literature (e.g., pre-registrations, open materials, data, and code).

On the theoretical level, more process-oriented research would be valuable - that is, research that directly examines the proposed mediating mechanisms by which mindfulness is proposed to foster prosocial behavior (improved executive control, self-regulation abilities and empathic concern). One possible way to address this is by using an experience sampling methodology in everyday life in the context of randomized controlled trials. Going beyond previous theorizing, we argue that the effects of increased mindfulness may not be inherently prosocial or anti-social. Instead, prosocial motivation may be required to increase prosocial behavior through increased mindfulness. Taken together, the hypothesis that mindfulness increases prosocial behavior is intriguing, but considerable work needs to be done both theoretically and empirically to convincingly demonstrate its validity and real-world ramifications. 


\section{References}

$(*)$ of special interest

$(* *)$ of outstanding interest

[1] Brown KW, Ryan RM, Creswell JD: Mindfulness: Theoretical foundations and evidence for its salutary effects. Psychol. Inquiry 2007, 18:211-237. https://doi.org/10.1080/10478400701598298

[2] Nilsson H, Kazemi A: Reconciling and thematizing definitions of mindfulness: The big five of mindfulness. Rev. General Psychol. 2016, 20:183-193. https://doi.org/10.1037/gpr0000074

[3] Van Dam NT, van Vugt MK, Vago DR, Schmalzl L, Saron CD, Olendzki A, Meissner T, Lazar SW, Kerr CE, Gorchov J, et al.: Mind the hype: A critical evaluation and prescriptive agenda for research on mindfulness and meditation. Persp. Psychol. Science 2016, 13:36-61. https://doi.org/10.1177/1745691617709589

[4] Bishop SR, Lau MA, Shapiro S, Carlson L, Anderson ND, Carmody J, Segal ZV, Abbey S, Speca M, Velting D, Devins G: Mindfulness: A proposed operational definition. Clinical Psychol. Science Practice 2004, 11:230-241. https://doi.org/10.1093/clipsy.bph077

[5] Brown KW, Ryan RM. The benefits of being present: Mindfulness and its role in psychological wellbeing. J. Pers. Soc. Psychol. 2003, 84:822-848. https://doi.org/10.1037/0022-3514.84.4.822

[6] Kabat-Zinn J: Full catastrophe living: Using the wisdom of your body and mind to face stress, pain and illness. Delacourt, 1990.

[7] Killingsworth MA, Gilbert DT: A wandering mind is an unhappy mind. Science 2010, 330:932-932. https://doi.org/10.1126/science.1192439

[8] Bargh JA, Chartrand TL: The unbearable automaticity of being. American Psychol. 1990, 54:462-479. https://doi.org/10.1037/0003-066X.54.7.462. 
[9] Friese M, Ostafin BD, Loschelder DD: Mindfulness as an intervention to improve selfcontrol. In Routledge international handbook of self-control in health and well-being. Edited by de Ridder D, Adriaanse M, Fujita K. Routledge; 2018:431-445.

[10] Schindler S: Ein achtsamer Blick auf den Achtsamkeits-Hype.

Organisationsberatung, Supervision, Coaching 2020, 27:111-124.

https://doi.org/10.1007/s11613-020-00641-z

[11] Monteiro LM, Musten RF, Compson J: Traditional and contemporary mindfulness:

Finding the middle path in the tangle of concerns. Mindfulness 2015, 6:1-13. https://doi.org/10.1007/s12671-014-0301-7

[12] Pfattheicher S, Nielsen YA, Thielmann I: Prosocial behavior and altruism: A review of concepts and definitions. Curr. Opinion Psychol. 2021. Advance Online Publication. https://doi.org/10.1016/j.copsyc.2021.08.021

[13] Batson C, Powell A: Altruism and prosocial behavior. In Handbook of psychology: Personality and social psychology. Edited by Millon T, Lerner MJ; John Wiley \& Sons, 2003:463-484. https://doi.org/10.1002/0471264385.wei0513.

[14] Schindler S, Reinhard MA: Catching the liar as a matter of justice: Effects of belief in a just world on deception detection accuracy and the moderating role of mortality salience. Pers. Individ. Diff. 2015, 73:105-109.

doi:10.1016/j.paid.2014.09.034

[15] Schindler S, Reinhard MA, Stahlberg D: Tit for tat in the face of death: The effect of mortality salience on reciprocal behavior. J. Exp. Soc. Psychol. 2013, 49:87-92. https://doi.org/10.1016/j.jesp.2012.06.002

[16] Cialdini RB, Brown SL, Lewis BP, Luce C, Neuberg SL: Reinterpreting the empathyaltruism relationship: When one into one equals oneness. J. Pers. Soc. Psychol. 1997, 73:481-494. https://doi.org/10.1037/0022-3514.73.3.481. 
[17] Penner LA, Dovidio JF, Piliavin JA, Schroeder DA: Prosocial behavior: Multilevel perspectives. Ann. Rev. Psychol. 2005, 56:365-392. https://doi.org/10.1146/annurev.psych.56.091103.070141.

[18] Creswell JD: Mindfulness interventions. Ann. Rev. Psychol. 2017, 68:491-516. https://doi.org/10.1146/annurev-psych-042716-051139

[19] Ostafin BD, Robinson MD, Meier BP: Handbook of mindfulness and self-regulation. Springer; 2015.

[20] Condon P: Meditation in context: Factors that facilitate prosocial behavior. Curr. Opinion Psychol. 2019, 28:15-19. https://doi.org/10.1016/j.copsyc.2018.09.011

[21] Donald JN, Sahdra BK, Van Zanden B, Duineveld JJ, Atkins PW, Marshall SL, Ciarrochi J: Does your mindfulness benefit others? A systematic review and metaanalysis of the link between mindfulness and prosocial behaviour. Brit. J. Psychol. 2019, 110:101-125. https://doi.org/10.1111/bjop.12338.

** This meta-analysis addressed correlational effects between trait mindfulness and prosocial outcomes as well as effects of mindfulness interventions on prosocial outcomes. Both analyses yielded a significant effect. Evidence for publication bias is reported.

[22] Berry, DR,Hoerr JP, Cesko S,Alayoubi A, Carpio K, Zirzow H, Walters W, Scram G, Rodriguez K, Beaver V. Does mindfulness training without explicit ethics-based instruction promote prosocial behaviors? A meta-analysis. Pers. Soc. Psychol. Bull. 2020, 46:1247-1269. https://doi.org/10.1177/0146167219900418.

** This meta-analysis addressed effects of mindfulness interventions on actual prosocial behaviors. Results show an overall significant effect. Evidence for publication bias is reported. 
[23] Pfattheicher S, Sassenrath C, Schindler S: Feelings for the suffering of others and the environment: Compassion fosters proenvironmental tendencies. Env. Beh. 2015, 48:929-945. https://doi.org/10.1177/0013916515574549

[24] Cohen J: Statistical power analysis for the behavioral sciences. Erlbaum; 1988.

[25] Schindler S, Pfattheicher S: When it really counts: Investigating the relation between trait mindfulness and actual prosocial behavior. Curr. Psychol. 2021. Advance online publication. https://doi.org/10.1007/s12144-021-01860-y

[26] Carter EC, Schönbrodt FD, Gervais WM, Hilgard J. Correcting for bias in psychology: A comparison of meta-analytic methods. Adv. Meth. Practices Psychol. Science 2019, 2:115-144. https://doi.org/10.1080/10478400701598298

[27] Kreplin U, Farias M, Brazil IA: The limited prosocial effects of meditation: A systematic review and meta-analysis. Scientific Rep. 2018, 8:2403. https://doi.org/10.1038/s41598-018-20299-z

[28] Luberto CM, Wasson RS, Kraemer KM, Sears RW, Hueber C, Cotton S: Feasibility, acceptability, and preliminary effectiveness of a 4-week mindfulnessbasedcognitive therapy protocol for hospital employees. Mindfulness 2017, 8:15221531. https://doi.org/10.1007/s12671-017-0718-X

[29] Funder DC, Ozer DJ: Evaluating effect size in psychological research: Sense and nonsense. Adv. Mett. Practices Psychol. Science 2019, 2:156-168. https://doi.org/10.1177/2515245919847202

[30] Friese M, Frankenbach J: P-hacking and publication bias interact to distort metaanalytic effect size estimates. Psychol. Meth. 2020, 25:456-471. https://doi.org/10.1037/met0000246

[31] Friese M, Hofmann W: State mindfulness, self-regulation, and emotional experience in everyday life. Motiv. Science 2016, 2:1-14. https://doi.org/10.1037/mot0000027 
[32] Fleeson W: Towards a structure- and process-integrated view of personality: Traits as density distributions of states. J. Pers. Soc. Psychol. 2001, 80:1011-1027. https://doi.org/10.1037/0022-3514.80.6.1011

[33] Böckler A, Tusche A, Schmidt P, Singer T: Distinct mental trainings differentially affect altruistically motivated, norm motivated, and self-reported prosocial behaviour. Scientific Rep. 2018, 8:13560. https://doi.org/10.1038/s41598-018-318138

[34] Singer T, Engert V: It matters what you practice: Differential training effects on subjective experience, behavior, brain and body in the ReSource Project. Curr. Opinion Psychol. 2019, 28:151-158. https://doi.org/10.1016/j.copsyc.2018.12.005.

[35] Ryan RM, Donald JN, Bradshaw EL: Mindfulness and Motivation: A process view using Self-determination theory. Curr. Directions Psychol. Science 2021, 30:300306. https://doi.org/10.1177/09637214211009511

[36] Poulin M, Ministero L, Gabriel S, Morrison C, Naidu E: Minding your own business? Mindfulness decreases prosocial behavior for those with independent selfconstruals. Psychol. Science in press. https://doi.org/10.31234/osf.io/xhyua * This article investigated and provided evidence for the idea that the effect of mindfulness on prosocial behavior depends on reported and salient self-construals (interdependence vs. independence).

[37] Schindler S, Pfattheicher S, Reinhard MA: Potential negative consequences of mindfulness in the moral domain. Europ. J. Soc. Psychol. 2019, 49:1055-1069. https://doi.org/10.1002/ejsp.2570

* This article points to a potential downside of mindfulness in the interpersonal context. In a series of five studies, evidence is provided that mindfulness attenuates repairing behavior that usually results from a bad conscience when having done harm. 